\title{
POLAR WANDERING AND MANTLE CONVECTION
}

\author{
H. TAKEUCHI and N. SUGI \\ Geophysical Institute, Faculty of Science, University of Tokyo, Japan
}

\begin{abstract}
According to the mantle convection theory, mantle materials come up to the surface of the Earth at the mid-oceanic ridge system, go off in two horizontal directions, and finally at the trench and orogenic belt system they return to the interior of the Earth. We assume no return flow in the deeper part of the mantle and calculate the change of products of inertia of the Earth due to the above mass transfer. The polar wandering thus calculated is towards the direction of about $90^{\circ}$ east and its absolute value is about $0.9 \times 10^{-2} \mathrm{~s} / \mathrm{yr}$.
\end{abstract}

It is well known that there is a secular wandering ot the pole of rotation. For example, according to N. Sekiguchi, as referred to in the book by Munk and MacDonald (1960), the speed of wandering is about $3 \times 10^{-3} \mathrm{~s} / \mathrm{yr}=1.4 \times 10^{-8} \mathrm{rad} / \mathrm{yr}$, and its direction is toward the longitude of Washington. The cause of this wandering is not yet clear. In the present paper, we propose the assumption that the polar wandering is due to mantle convection. According to the mantle convection theory, mantle materials come up to the surface of the Earth at the mid-oceanic ridge system, go off in two horizontal directions, and finally at the trench and orogenic belt system they return to the interior of the Earth. Concerning the direction of mantle convection on the Earth's surface, and the distribution of the ridge and trench-orogenic belt system, see, for example, Wilson's paper (1967).

We suppose here that in the deeper part of the mantle, there is no return flow to cancel the flow on the Earth's surface. Then it follows that some amount of the mantle materials disappears from the oceanic ridge system with time, and the same amount of materials accumulates in the trench-orogenic belt system. In view of the fact that deep focus earthquakes do not occur in the mantle at depths below $700 \mathrm{~km}$, our assumption of no return flow does not seem too unlikely. This assumption, once being admitted, will give secular changes of the products of inertia of the Earth and will lead to the secular wandering of the pole. We now estimate the amount of this wandering.

First of all, we estimate the amount of mantle materials coming up at the oceanic ridge system. The total length of the ridge system is about $65000 \mathrm{~km}$. Suppose that its width is $200 \mathrm{~km}$ and the speed of mantle convection is $3 \mathrm{~cm} / \mathrm{yr}=3 \times 10^{-5} \mathrm{~km} / \mathrm{yr}$, and the total volume coming up is $65000 \times 200 \times 3 \times 10^{-5}=390 \mathrm{~km}^{3}$ per yr. If the density of mantle material is $3 \mathrm{~g} / \mathrm{cm}^{3}$, the corresponding total mass $m$ will be about $1.2 \times 10^{18} \mathrm{~g} / \mathrm{yr}$. The same amount of materials accumulates in the trench-orogenic belt system. For the approximate estimate we are now making, it seems proper to distribute these materials uniformly over the ridge and trench-orogenic belt system.

It may be interesting to predict the direction of the polar wandering before our detailed calculation. Generally speaking, the pole should drift from the trenchorogenic belt system and toward the ridge system. The ridge system distribution is 
fairly uniform on the Earth's surface; and on the contrary the trench-orogenic belt system is concentrated around the Pacific ocean and the Himalaya-Alps. In view of this fact, we might think that the pole would drift toward the east coast of North America. As we have mentioned before, this agrees well with the observed direction of wandering.

In calculating changes of the products of inertia, we use the following orthogonal coordinate system. The origin of the system is at the center of the Earth, the $x$ and $y$ axes are in the directions of the longitude of Greenwich and $90^{\circ}$ east respectively, and the $z$ axis is in the direction which together with the above $x$ and $y$ axes, will make a right-handed system. This coordinate system is the same as that adopted by Munk and MacDonald in their book. But in drawing the polar motion curve, we usually direct the $y$ axis to $90^{\circ}$ west. Now according to our calculations, the changes in the products of inertia, $C_{x z}$ and $C_{y z}$, are as follows:

ridge system: $C_{x z}=-0.015 m R^{2}, \quad C_{y z}=-0.096 m R^{2}$

trench and orogenic belt system:

$$
\begin{array}{ll} 
& C_{x z}=0.037 m R^{2}, \quad C_{y z}=-0.13 m R^{2} \\
\text { total : } & C_{x z}=0.022 m R^{2}, \quad C_{y z}=-0.22 m R^{2} .
\end{array}
$$

We made these calculations for the ridge system and the trench-orogenic belt system independently, and summed up the results afterwards. As mentioned before, $m$ is the total mass of materials coming up at the ridge system per year, and this is equal to that going down at the trench-orogenic belt system. This $m$ is equal to $1.2 \times 10^{18} \mathrm{~g} / \mathrm{yr}$, and $R$ is the radius of the earth. On the other and, according to the book by Munk and MacDonald (1960), the amounts of polar wanderering $\phi_{x}$ and $\phi_{y}$ in the $x$ and $y$ directions are as follows.

$$
\phi_{x}=\frac{C_{x z}}{C-A}, \quad \phi_{y}=\frac{C_{y z}}{C-A} .
$$

Here $C$ is the moment of inertia around the $z$ axis, and $A$ that around the $x$ or $y$ axis. Making use of

$$
\frac{1}{C-A}=\frac{300}{C}, \quad C=0.33 M R^{2}, \quad \begin{aligned}
& m \\
& M
\end{aligned}=2 \times 10^{-10}
$$

we have

$$
\phi_{x}=4.1 \times 10^{-9}, \quad \phi_{y}=-4.1 \times 10^{-8} .
$$

The result show that the polar wandering is toward the direction of about $90^{\circ}$ west, and its absolute value is about $4.1 \times 10^{-8} \mathrm{rad} / \mathrm{yr}=9 \times 10^{-3} \mathrm{~s} / \mathrm{yr}$. Thus the calculated direction of wandering agrees well with that observed and its absolute value is about three times as large as the observed value. This difference in the absolute values can be removed by assuming the return flow in the deeper part of the mantle to be of magnitude equal to two thirds of the main flow. 


\section{References}

Munk, W. H. and MacDonald, G. J. F.: 1960, The Rotation of the Earth, Cambridge University Press, p. 176.

Wilson, J. T.: 1967, in T. F. Gaskell (ed.), The Earth's Mantle, Academic Press, New York and London.

\section{DISCUSSION}

P. Melchior: What is the depth of return flow necessary to adjust the calculated figure to the observed drift of $0.003^{\prime \prime} / \mathrm{yr}$ ?

H. Takeuchi: $1000 \mathrm{~km}$.

P. Melchior: Is that not in contradiction with the fact that there are no earthquakes deeper than $700 \mathrm{~km}$ ?

H. Takeuchi: This is only an order of magnitude.

P. Melchior: You have also to take account of the ice cap melting. As this gives also a drift in the Washington meridian direction. You should have a greater depth of the return flow.

H. Takeuchi: That is correct.

P. Melchior: You suggest very large convection cells in the upper mantle is that really possible? H. Takeuchi: There should be 2 or 3 convection cells.

Nakai: In some of island arc and trench systems, including the Japanese Islands and the Japan trench, the plus and minus regions of free air anomalies of gravity are almost equal in area and in magnitude. This fact shows there is no excess of mass as a whole. I do not think that the accumulation of mantle materials occurs.

H. Takeuchi: I mentioned not the mass excess of static state, but the rate of mass accumulation.

Cheh Pan: Whether the directions of the secular polar motion coincides with the longer axis of the ellipse described by the periodic components of the polar motion?

H. Takeuchi: No relation.

Cheh Pan: What is the lateral dimension of the convection?

H. Takeuchi: Thousands of kilometers.

Cheh Pan: The melting of ice caps is a result of the secular polar motion rather than the cause, since the change of products of inertia due to the melting of ice caps is too small to have any effect on the stability of the earth's rotation.

$M$. A. Chinnery: A steady state system of convection can not give a secular polar drift.

E. M. Gaposhkin: Did you say that a shift of the pole would occur even with complete mass return at depth?

H. Takeuchi: (after discussion) I agree. My calculation is not valid if there is complete return current. 